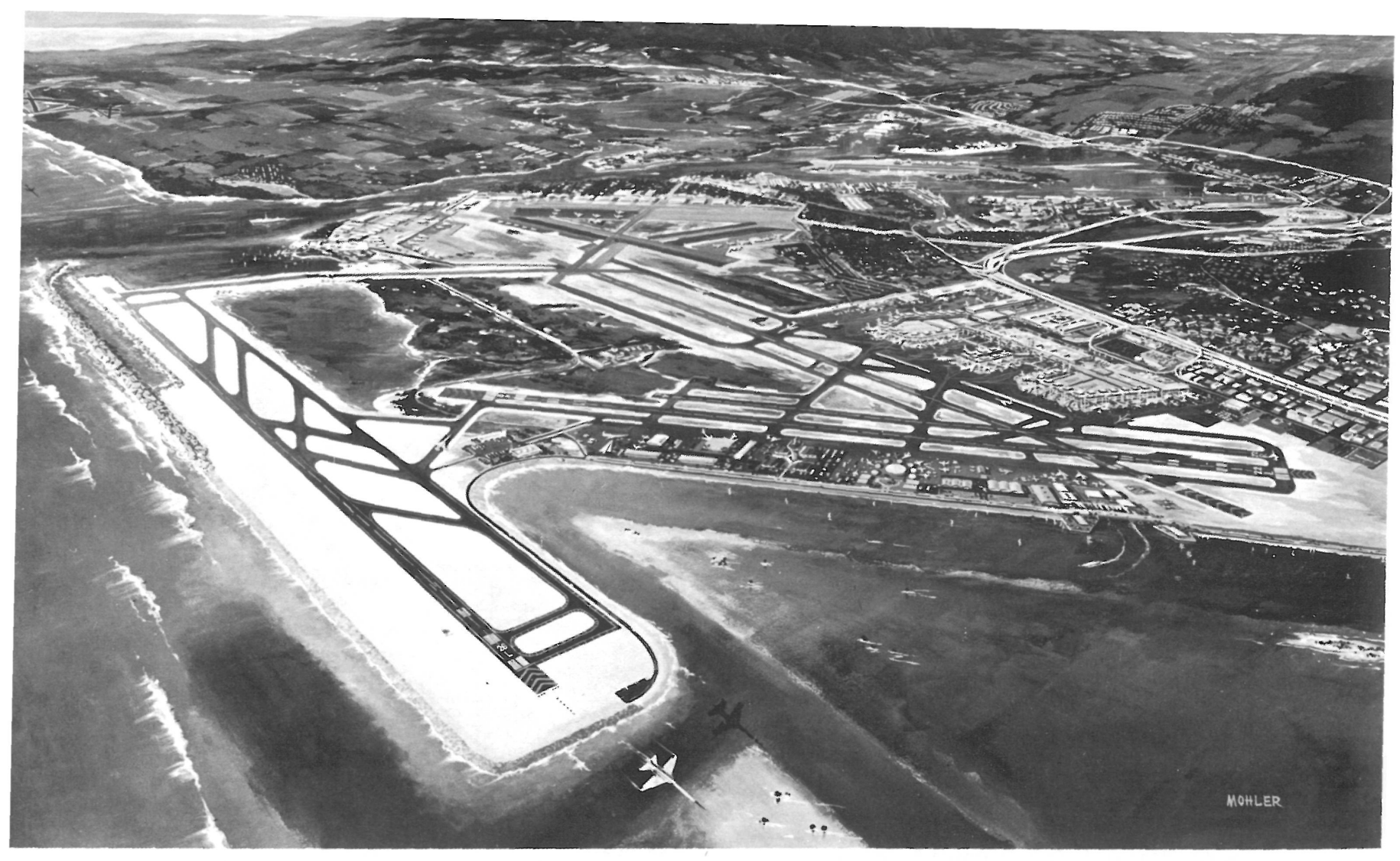

\title{
Pista de aterrizaje sobre un arrecife de coral EE.UU.
}

Ralph M. Parsons, Cía., ingenieros y constructores

sinopsis

$541-21$

\begin{abstract}
Probablemente, la mayor obra de ampliación hacia el mar realizada hasta ahora de un aeropuerto se ha hecho en el Aeropuerto Internacional de Honolulú, Hawai, donde se ha construido una pista de aterrizaje de $3.600 \mathrm{~m}$ de longitud, sobre un arrecife de coral.

Esta obra, difícil y única en su género, abre nuevas posibilidades para que los aeropuertos puedan solucionar sus problemas de capacidad y ambientales mediante la ampliación marítima.
\end{abstract}

\section{Introducción}

Se ha realizado en el Aeropuerto Internacional de Honolulú una nueva pista de aterrizaje con su dique de protección y sus pistas de rodaje complementarias. La pista de $3.600 \mathrm{~m}$ está situada sobre un arrecife de coral. La Isla de Oahu, zona del proyecto, es el centro de la industria turística de Hawai, la cual proporciona anualmente varios millones de dólares a Hawai. Se ha conseguido, de esta manera, un aumento de capacidad muy necesitado por el aeropuerto, además de una disminución del ruido y un incremento de la seguridad en las zonas céntricas de Honolulú y en sus suburbios.

Debido al poco terreno disponible para la ampliación del aeropuerto tierra adentro, la obra se ha construido sobre 14,50 millones de metros cúbicos de coral dragados y colocados en un arrecife de coral ya existente.

La nueva pista se ha situado paralela y a $1.800 \mathrm{~m}$ hacia el sur de la pista actual (la 8L-26R), de $3.775 \mathrm{~m}$, y enlaza con la red existente mediante nuevas pistas de rodaje. El complejo formado por las pistas de aterrizaje y rodaje está protegido del mar por un dique de $4.800 \mathrm{~m}$ de longitud. 


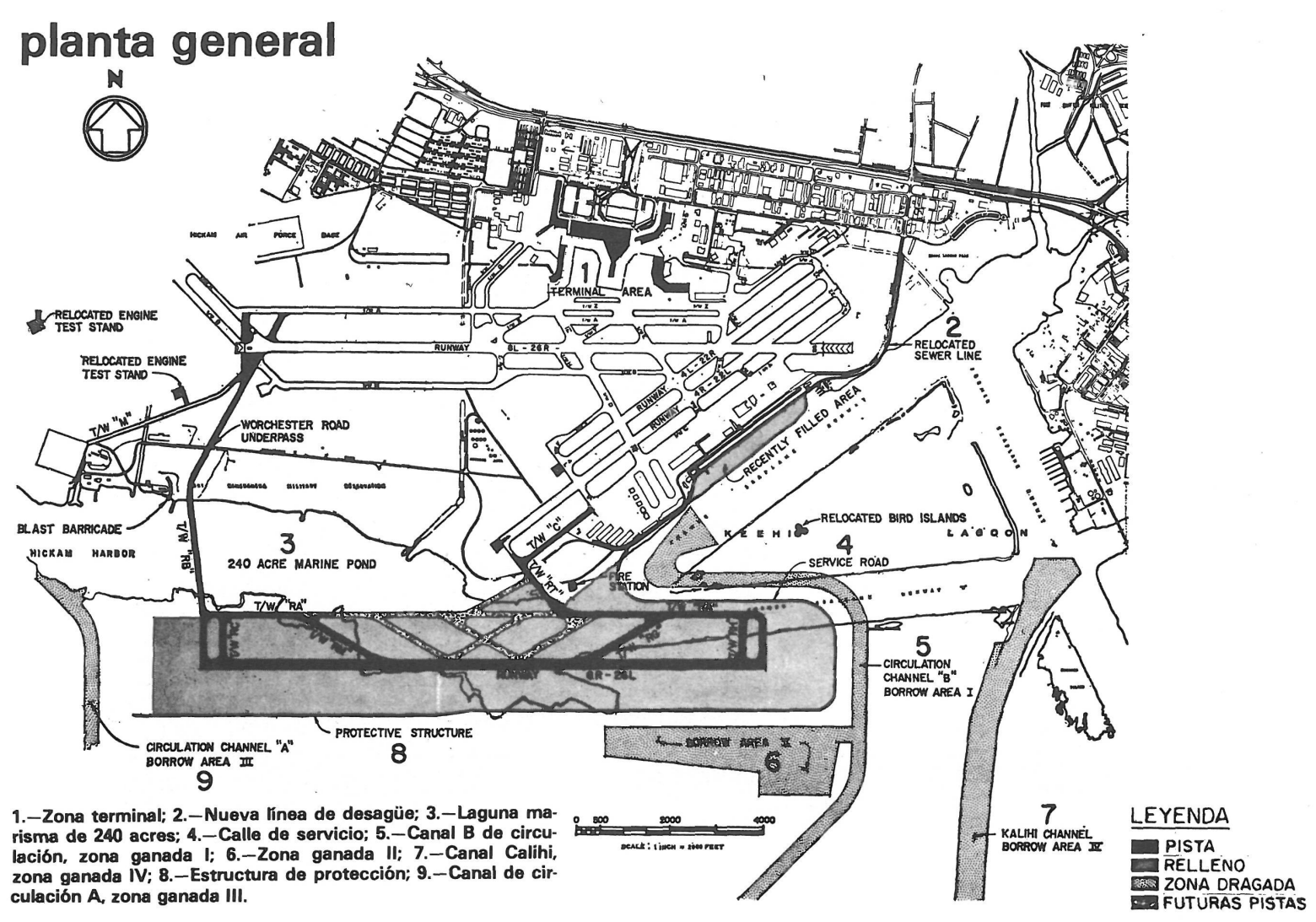

El Aeropuerto Internacional de Honolulú está situado a aproximadamente $8 \mathrm{~km}$ del centro de la Ciudad. Realizado en 1927, se unió en 1957 a la contigua Base Aérea de Hickham, mediante el enlace de las dos pistas de aterrizaje principales, con lo que se consiguió una pista de $3.775 \mathrm{~m}$ de longitud. Desde entonces el aeropuerto es utilizado conjuntamente por la U.S. Air Force y las líneas civiles aéreas.

Los estudios realizados, desde 1956, con objeto de solucionar los problemas del ruido y de los vuelos sobre las zonas más densas de la ciudad llevaron a la conclusión, en 1968, de que era necesaria una nueva pista de aterrizaje al sur de la existente.

En efecto, las operaciones de vuelo realizadas en el aeropuerto durante 1973 fueron 309.114, existiendo la previsión de que serán 439.000 en 1980 y 546.300 en 1990 . La capacidad horaria del aeropuerto, antes de la realización de la nueva pista, estaba entre 76 y 82 movimientos, con dos horas al día de una gran sobrecarga de tráfico. La nueva pista aumentará la capacidad punta horaria entre 112 y 119 operaciones.

\section{Estudios ingenieriles y del medio ambiente}

Entre los trabajos realizados están: estudios hidrográficos y topográficos, fotografias aéreas, estudios planimétricos, estudios para determinar la capacidad de la cimentación y de los materiales de relleno y la obtención de los datos geológicos en toda la zona, así como la evaluación del entorno oceánico que puede tener influencia en el proyecto y construcción.

En la Universidad de Hawai se construyó un modelo hidráulico tridimensional para examinar los efectos de las olas de los temporales sobre la estructura protectora de la pista. El modelo realizado a las escalas: horizontal 1:600 y vertical 1:200, incluía las zonas adyacentes hasta una distancia de, aproximadamente, $16 \mathrm{~km}$.

Otros modelos hidráulicos bidimensionales se hicieron para comprobar los criterios de diseño de la estructura de protección y para determinar la altura de las olas y la sección transversal óptima de dicha estructura. Las corrientes del océano y de la laguna interior, y el estado ecológico de esta laguna y del arrecife se estudiaron al detalle. También se hicieron estudios exhaustivos sobre la vida de los pájaros y de los peces que habitan la zona de la laguna y el arrecife. 


\section{Condiciones del emplazamiento}

Existen tres superficies perfectamente diferenciadas en la zona: la situada en el mar, próxima al arrecife; la plana, propia del arrecife; y la superficie excavada.

El material de superficie de la zona próxima al arrecife, pero situada en aguas profundas y de cierto relieve, lo componen pequeños fragmentos de coral que sobresalen del terreno, fragmentos mayores, gravas y arenas de variado espesor.

El material de la superficie plana del arrecife, situada al exterior 0 en aguas poco profundas, se caracteriza por estar formado a base de corales muertos cubiertos de algas y piedras de coral con una costra dura y delgada de unos $30 \mathrm{~cm}$ de espesor. Esta costra se retiró de las áreas de seguridad de las pistas de aterrizaje y rodaje, y se utilizó en la parte menos profunda de la estructura de protección.

Las superficies excavadas que varian en espesor desde $-3,60$ a $-4,50 \mathrm{~m}$, bajo el nivel del mar, en la parte destinada a pistas, hasta $-9 \mathrm{~m}$, en algunas áreas de préstamo, tienen en el fondo unos sedimentos fangosos compresibles y muy blandos de 0,60 a $4,50 \mathrm{~m}$ de espesor. Estos sedimentos se quitaron antes de volver a colocar los rellenos de coral previamente dragados.

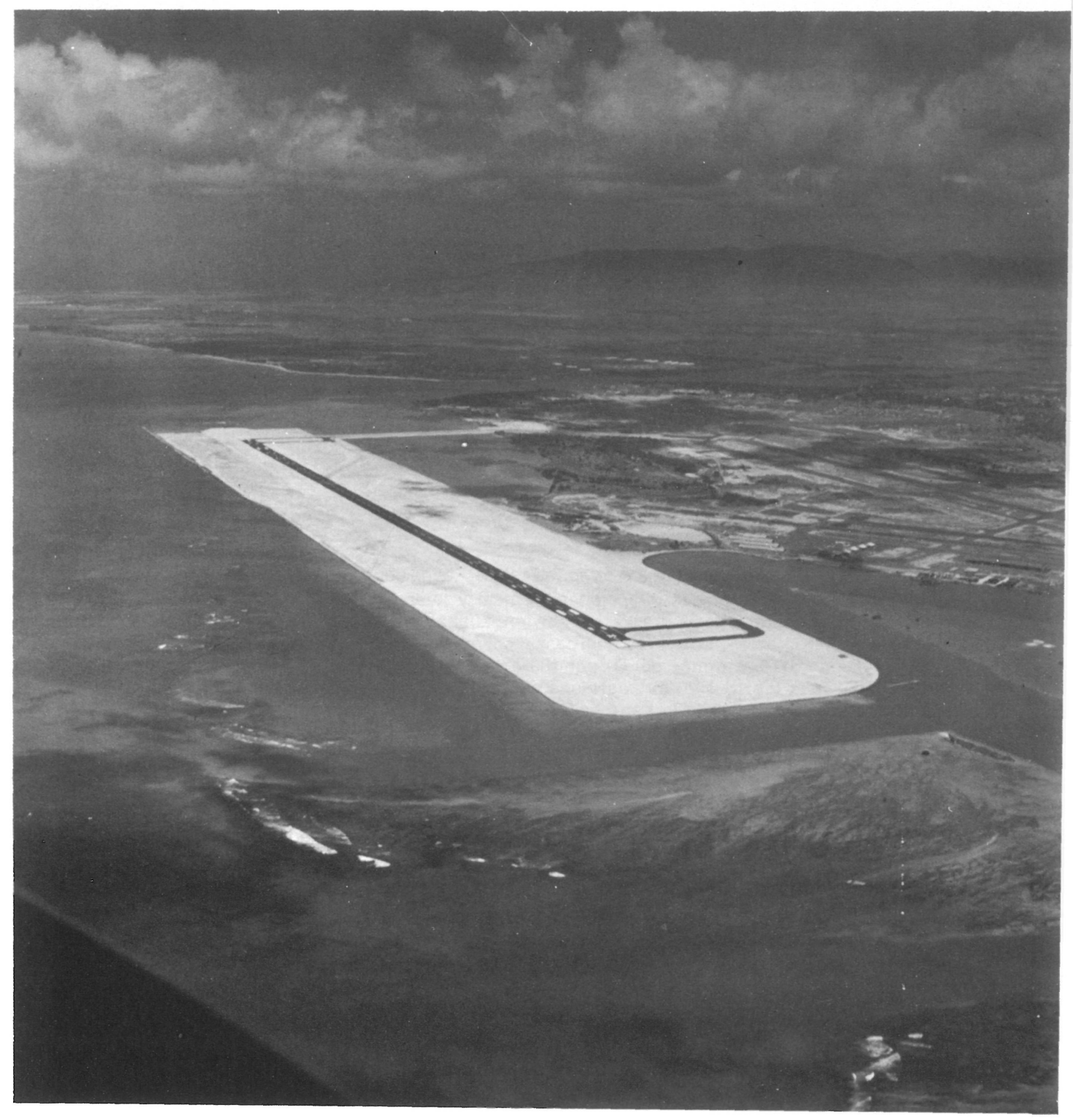




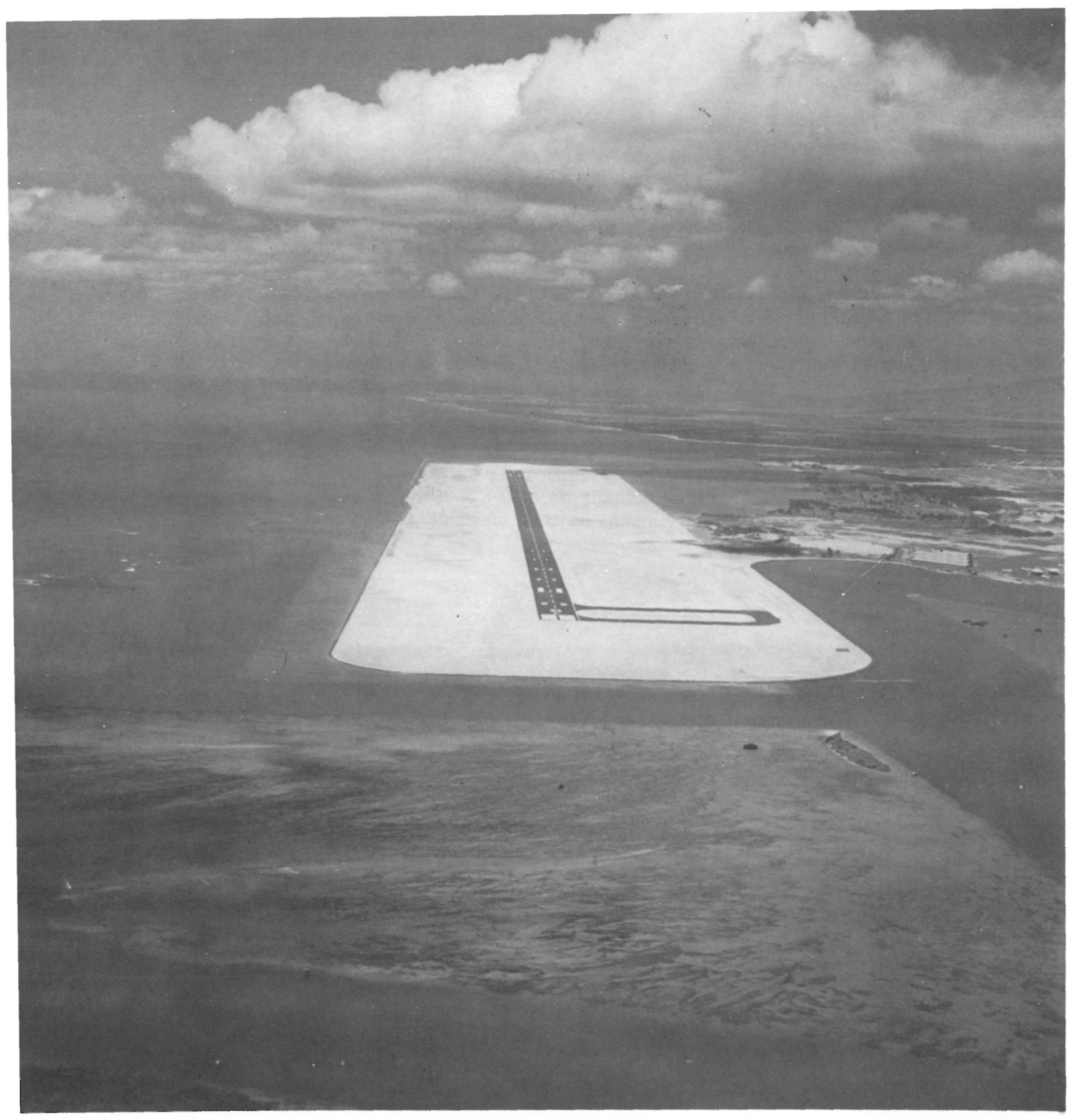

Como es típico de la mayoría de los arrecifes, el de Keehi tiene una estructura y una distribución de sedimentos heterogénea en las direcciones: vertical y lateral. Los primeros 9 a 10,50 m del arrecife están formados por arenas y gravas de aluvión sueltas y, en ocasiones, cementadas además de cabezas de coral intactas. Los finos que pasan por el tamiz $n .^{\circ} 200$ de la serie U.S. Standard varian desde un 10 por 100 hasta más del 50 por 100 en arcillas y zonas de fango. Entre los 9 ó 10,50 m de profundidad, hasta los 18 ó $20 \mathrm{~m}$ existe una caliza bastante cementada. Debajo de esta zona hay una capa de material similar al de la parte superior.

\section{Proyecto}

La pista de aterrizaje tiene $3.600 \mathrm{~m}$ de longitud, $60 \mathrm{~m}$ de anchura y una altura media de $3 \mathrm{~m}$ sobre el nivel del mar, con áreas de seguridad de $105 \mathrm{~m}$ de anchura y zonas sin obstáculos, de $300 \mathrm{~m}$, a ambos lados del eje longitudinal de la pista. En los extremos de la pista hay unos $30 \mathrm{~m}$ más para algún caso imprevisto. Las pistas de rodaje tienen una anchura de $30 \mathrm{~m}$, con áreas de seguridad a ambos lados de un ancho mínimo de $33 \mathrm{~m}$ y un máximo de $45 \mathrm{~m}$. El eje longitudinal de la pista de rodaje, paralela a la de aterrizaje, está a una distancia de $315 \mathrm{~m}$ del eje longitudinal de ésta. 
El pavimento de la pista de aterrizaje consiste en $12,50 \mathrm{~cm}$ de hormigón asfáltico colocados sobre una capa base de árido machacado de $45 \mathrm{~cm}$ de espesor. Debido a la posibilidad de asientos, especialmente en las zonas donde las pistas de aterrizaje y de rodaje pasan sobre zonas previamente dragadas, hay previsiones para, en los cinco años siguientes a la construcción, agregar un hormigón asfáltico de $12,5 \mathrm{~cm}$ de espesor, con objeto de corregir dichos asientos e incrementar la resistencia del pavimento para mayores cargas.

Los materiales de coral dragados, que se colocan debajo del pavimento de las pistas, están clasificados como relleno estructural y no pueden tener más del 15 por 100 de finos que pasan por el tamiz n. 200 U.S. Standards. Los demás materiales dragados se clasifican como relleno común y se colocan fuera de las zonas de relleno estructural. El material que se usa como relleno estructural, después de hacer pasar sobre él un rodillo de $60 \mathrm{t}$, se coloca en capas de $20 \mathrm{~cm}$ y se compacta al 95 por 100 de la densidad máximá en la parte superior del terreno. De las seis pistas de alta velocidad sólo dos han sido pavimentadas en esta primera fase: una en el lado este y otra en el oeste.

El drenaje de la zona situada entre la pista de aterrizaje y el dique de protección se hace por un canal abierto en el extremo oeste. El drenaje entre la pista de aterrizaje y las pistas de rodaje se hace por conductos de hormigón que tienen sus conductos de salida sumergidos a fin de procurar los desagües del colector. El flujo hacia adentro y hacia afuera del estanque marino de 100 ha, situado entre las pistas de rodaje y el contorno de la superficie del agua, se realiza por 10 tubos de hormigón de $180 \mathrm{~cm}$ de diámetro. Un tubo de $180 \mathrm{~cm}$ va por debajo de la estructura de protección, las pistas de rodaje y la pista de aterrizaje, y enlaza el estanque marino con el océano. Si fuese necesario, el agua del océano puede ser bombeada por este conducto.

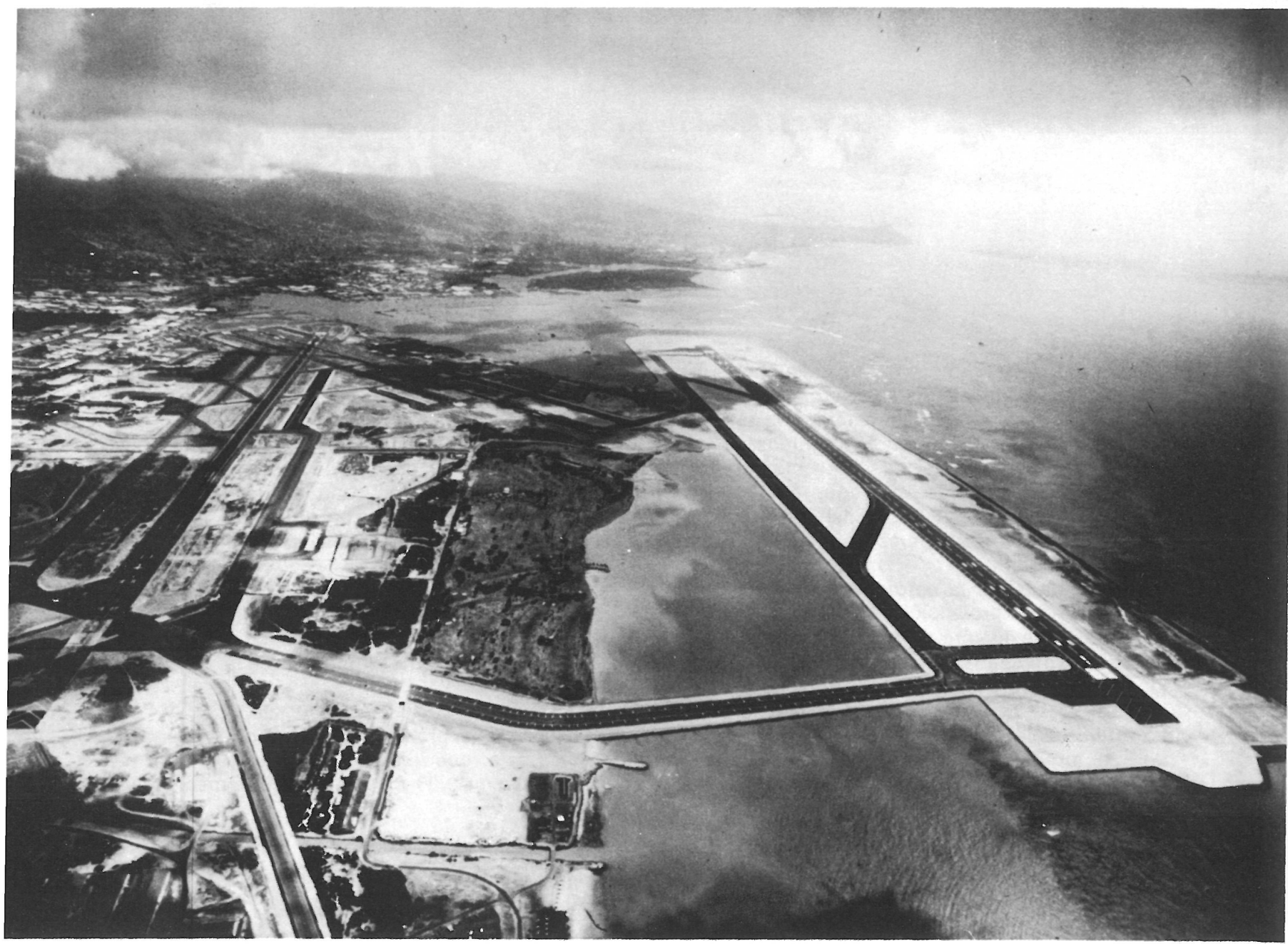




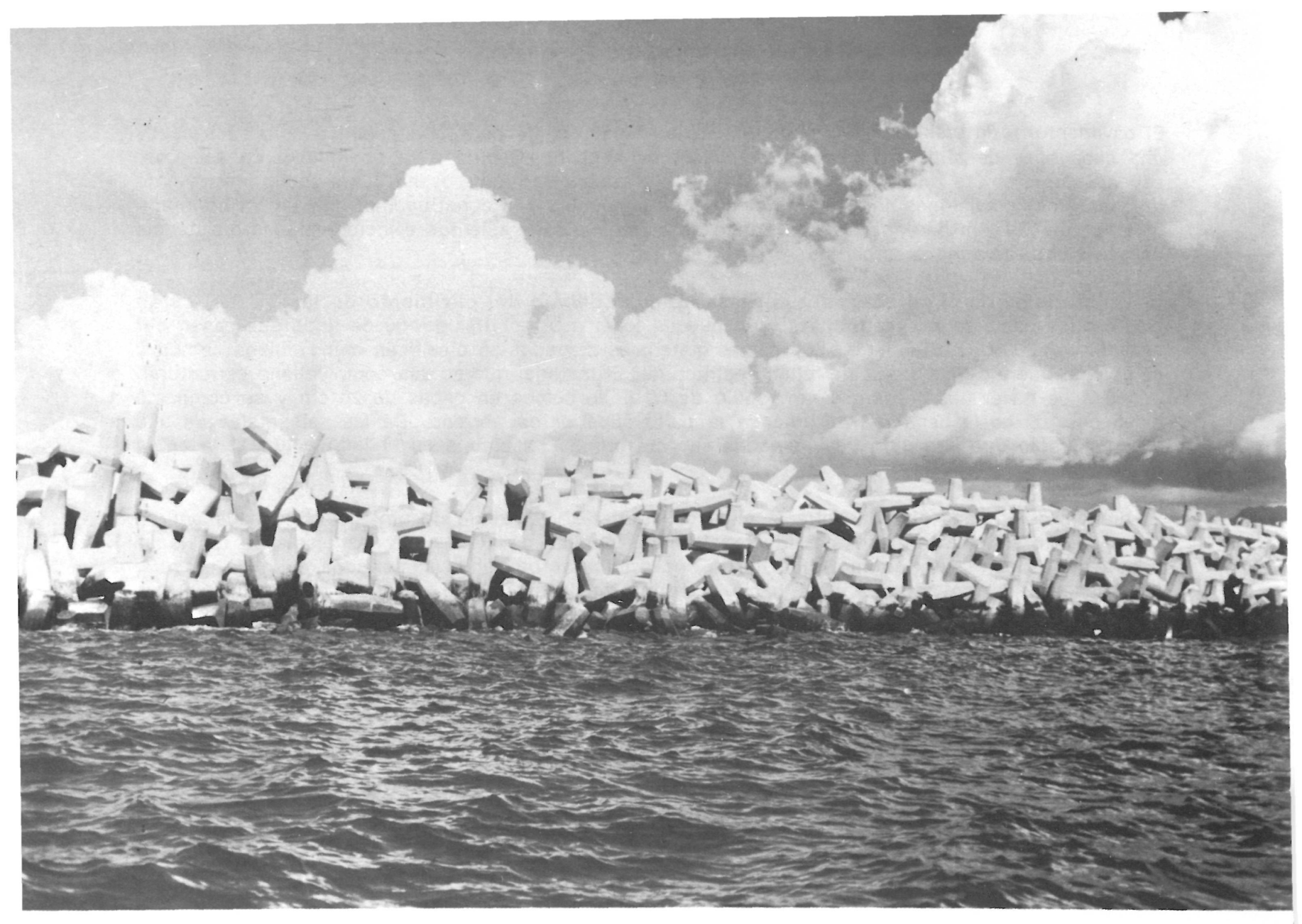

La estructura de protección de la pista de aterrizaje, de $4.800 \mathrm{~m}$ de longitud, es paralela al eje longitudinal de dicha pista en su mayor parte describiendo un giro de $90^{\circ}$ en su extremo este y cruzando el arrecife para empalmar con el camino de servicio, el cual da acceso al complejo pista de aterrizaje/pistas de rodaje y los equipos de operaciones y de mantenimiento. El dique de protección tiene una altura variable, desde un mínimo de $1,20 \mathrm{~m}$, hasta un máximo de $6 \mathrm{~m}$ sobre el nivel medio del mar. La profundidad del agua en el pie de la estructura de protección varía desde $0 \mathrm{~m}$ hasta $-8,10 \mathrm{~m}$ bajo el nivel medio del mar. La parte superior del dique se basa en la altura de las olas, determinada por estudios sobre modelo hidráulico y sobre estructuras prototipo.

Una gran parte de la estruciura está situada detrás de un arrecife poco profundo, lo que le da un cierto grado de protección natural, mientras que $2.100 \mathrm{~m}$ se encuentran expuestos a la acción directa del oleaje. En cuanto a las alturas de las olas previstas, se considera que las olas de 4,50 $\mathrm{m}$ se producirán frecuentemente; las de $6 \mathrm{~m}, 1$ - a 10 horas al año; las de $7,50 \mathrm{~m}$, una vez cada 10 años; siendo las de $9 \mathrm{~m}$ posibles pero muy improbables.

La altura de la marea más alta recordada en Honolulú Harbor, zona próxima a la Laguna Keehi, es de 0,72 m sobre el nivel medio del mar, mientras que la más baja, ocurrida en 1911 , es de $-0,60 \mathrm{~m}$ bajo el nivel medio del mar. Las alturas medias de las mareas son $0,30 \mathrm{~m}$ sobre el nivel medio del mar y $-0,24 \mathrm{~m}$ bajo el nivel medio del mar.

En el proyecto se consideró, para el Estanque Keehi, una sobreelevación del agua debida al viento igual a $0,21 \mathrm{~m}$. Esta altura, que es una condición extrema, sumada a la altura máxima de marea de $0,72 \mathrm{~m}$ nos da una altura de $0,90 \mathrm{~m}$, que fue la considerada en el proyecto y que es muy conservadora. 
De las cinco posibles alternativas para la parte profunda de la estructura de protección:

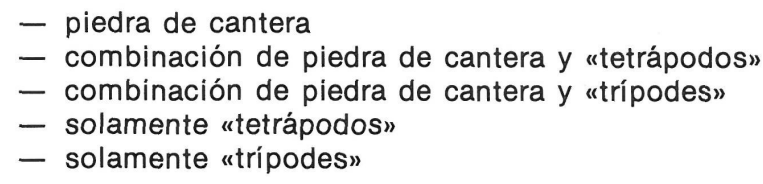

se eligió la solución en la que sólo se emplean "tetrápodos", que tiene una gran estabilidad. Se emplearon 14.010 "tetrápodos" de 4 t y 4.650 de 6 t.

Además de los proyectos de las pistas de aterrizaje, las pistas de rodaje, pavimentación y alumbrado, los pasos subterráneos de vehículos y el nuevo trazado de la red de alcantarillado, se han hecho los de una nueva zona recreativa para la U.S. Navy, los de dos nuevas zonas de habitat para pájaros, y los de una nueva estación de bomberos.

Existe un plan, para el uso preferencial de esta nueva pista por los aviones accionados por turbina, con el que se pretende disminuir el ruido y asegurar el uso adecuado del campo en la zona que rodea al aeropuerto. Este plan impone pesos y tiempos máximos de despegue en la antigua pista $8 \mathrm{~L}-26 \mathrm{R}$, desviando el tráfico hacia la nueva pista. De esta forma se obtendrá una reducción del ruido de 20 decibelios.

\section{Construcción}

El plan de obra propuesto para construir la nueva pista de aterrizaje, incluía terraplenes de confinamiento colocados de modo que no impidiesen la realización de la obra y, a la vez, guardasen la mayor cantidad posible de materiales. El material de coral dragado, usado como relleno, se bombeó hasta estos terraplenes, desde las cuatro áreas de préstamo, mediante dragas de succión hidráulica. Una vez terminada esta fase, los 14,50 millones de metros cúbicos se bombearon a la zona del arrecife, donde se hicieron la pista de aterrizaje y las de rodaje. La draga de succión hidráulica usada para estos trabajos es la Hidro-Pacific de $90 \mathrm{~cm}$, accionada eléctricamente. Con ella se consiguió una media de $19.000 \mathrm{~m}^{3} / \mathrm{día}$, con una producción diaria máxima de $33.250 \mathrm{~m}^{3}$. Tres tubos de $90 \mathrm{~cm}$ de diámetro colocados transversalmente a la zona de la pista de aterrizaje sirvieron para descargar el relleno en el borde de las zonas que se iban a pavimentar. Igualmente dos tubos de $90 \mathrm{~cm}$ sirven para colocar el rellano estructural en las pistas de rodaje.

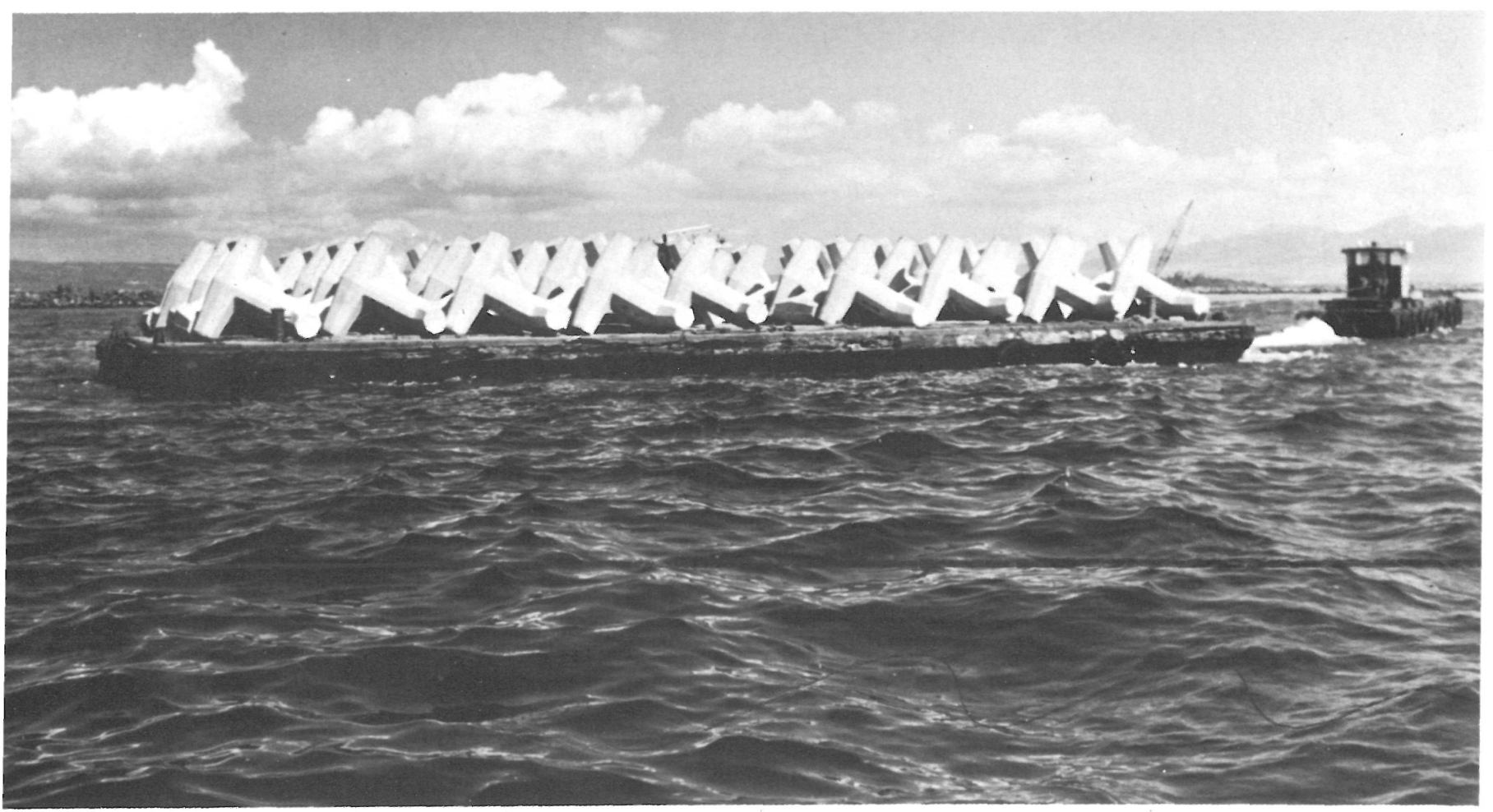


La draga de $40 \mathrm{~cm}$, Explorer, quitó el lodo blando, altamente compresible, situado en las zonas previamente dragadas donde se iba a realizar la pavimentación y bombeó este material a las zonas de vertido, situadas fuera de las áreas de seguridad de las pistas donde se cubrieron con 0,9 a $1,20 \mathrm{~m}$ de material de coral dragado. Para impedir el desmoronamiento en las zanjas de fango dragadas, éstas se rellenaron hasta una cierta altura antes de la colocación del relleno estructural.

El material de relleno estructural que se usó en las zonas del pavimento se llevó, primero, hasta una altura de $0,90 \mathrm{~m}$ por medio de scrapers provistos de neumáticos de caucho, y luego se pasó sobre él un rodillo con neumáticos de caucho de $60 \mathrm{t}$. Las irregularidades, roderas, puntos blandos 0 depresiones desarrollados durante la compactación se corrigieron mediante disgregación, aportación, eliminación o reemplazo de material hasta que se consiguió una superficie lisa y uniforme. Después de esta compactación, el material se colocó en capas de $20 \mathrm{~cm}$ de espesor mediante scrapers y se compactó hasta un 95 por 100 de densidad máxima usando un rodillo de pie de cabra y un rodillo vibratorio de rueda lisa.

El núcleo de la parte menos profunda de la estructura de protección se realizó con piedra viva de coral recogida del arrecife situado en el extremo este de la obra. La piedra de coral fue primero escarificada con un Ripper D-9 y llevada después por una grúa provista de cucharón de arrastre.

El núcleo de la parte más profunda de la estructura sólo tiene el 10 por 100 del material inferior a los $12 \mathrm{~cm} \mathrm{y}$, como mínimo, el 10 por 100 superior a $45 \mathrm{~cm}$. Este material del núcleo, el material entre capas y el material de armadura es de andesita y basalto obtenidos de una cantera que hay en la Isla de Molokai, situada a, aproximadamente, $90 \mathrm{~km}$ de Oahu.

Para transportar y colocar el material en la parte más profunda de la estructura de protección se usaron barcazas de $800 \mathrm{t}$ basculables hidráulicamente y barcas de $2.000 \mathrm{t}$ con grúas flotantes colocadas sobre ellas. Se necesitaron $700.000 \mathrm{t}$ de piedra para construir la estructura.

Los "tetrápodos" se hicieron en una explanada situada en el extremo este de la obra, se trasladaron sobre balsones de fondo plano y se colocaron con grúas flotantes por la parte del mar y con grúas móviles por la parte superior de la estructura.

Durante la construcción se tomaron las adecuadas medidas de precaución para proteger el medio ambiente.

WAYNE L. RICKERD

\section{résumé}

PISTE D'ATTERRISSAGE SUR UN RECIF DE CORAUX

Etats-Unis

Ralph M. Parsons, Cie., ingénieurs et constructeurs

Probablement, les travaux d'agrandissement les plus importants qui aient été jusqu' présent réalisés dans un aéroport côtier ont été ceux de l'aéroport international de Honolulu, Hawaii, où une piste d'atterrissage de $3.600 \mathrm{~m}$ de long a été exécutée su un récif de coraux.

Cet ouvrage, difficile et unique dans son genre, ouvre de nouvelle perspectives pour que les aéroports puissent résoudre leurs problèmes de capacité et d'environnement grâce à des travaux d'agrandissement maritimes.

\section{summary \\ LANDING STRIP ON A CORAL REEF U.S.A.}

Ralph M. Parsons \& Co.

Engineers and Builders

This is probably the largest land reclaim project ever attempted to enlarge an airport facility. The owner is the Honolulu International Airport, Hawaii, where a $3,600 \mathrm{~m}$ long landing strip was built on a coral reef.

This project, difficult and unique, opens new ways for airports to solve their capacity and environment problems through reclaiming land from the ocean.

\section{zusammenfassung \\ LENDEPISTE AUF EINEM KORALLENRIFF - USA}

Ralph M. Parsons, Ingenieure und Konstrukteure

Die bisher wahrscheinlich grössten Erweiterungsarbeiten zum Meer hin wurden auf dem Internationalen Flughafen von Honolulu, Hawai, durchgeführt, wo man eine $3.600 \mathrm{~m}$ lange Landepiste auf einem Korallenriff baute.

Diese schwierige, in ihrer Art einzigartige Arbeit öffnet den Weg zu neuen Möglichkeiten, welche es den Flughäfen erlauben, ihre Probleme im Zusammenhang mit ihrem Fassungsvermögen und ihrer Umgebung zu lösen, indem Erweiterungen über dem Wasser vorgenommen werden. 